\title{
TRATAMENTO MICROCIRÚRGICO DE ANEURISMAS DA ARTÉRIA COMUNICANTE ANTERIOR
}

\author{
Luiz Carlos Mendes Faleiro * \\ Pedro Alcantara Rodrigues * \\ JosE ANTONIO RODRIGUES * \\ ITAMar Megda Cesarini ** \\ ANTONIO Sergio BonfiM ***
}

A artéria comunicante anterior é a sede mais freqüente de aneurismas saculares atingindo a $30 \%$ do total ${ }^{20}$, sendo mais comum no sexo masculino. A etiopatogenia destas dilatações arteriais ainda não está completamente esclarecida. Enquanto alguns autores citam defeitos congênitos na parede arterial criando locais de menor resistência, outros afirmam haver evidência de degeneração da camada elástica em conseqüência de fatores hemodinâmicos. A desigualdade de calibre entre as porções proximais das artérias cerebrais anteriores ocorre significativamente nos aneurismas desta localização, sendo que o enchimento de ambas as artérias cerebrais anteriores se faz a custa de uma das carótidas, transformando a artéria comunicante anterior em verdadeira e ativa bifurcação arterial. Anatomicmeante situado próximo a ramos que nutrem o hipotálamo e lobos frontais a rotura ocasiona sintomas psíquicos de duração variável.

A história natural destes aneurismas ${ }^{17,20}$ mostra que no primeiro ano após a rotura a mortalidade é de $64 \%$ e que $25 \%$ dos sobreviventes encontramse parcial ou totalmente incapacitados. A mortalidade decorre principalmente de novas roturas, as quais são mais freqüentes nas duas primeiras semanas após a primeira hemorragia subaracnóidea.

O tratamento cirúrgico destes aneurismas é considerado como tarefa das mais dificeis, só sendo suplantada pelos aneurismas da bifurcação da artéria basilar que, no entanto, ocorrem com muito menor freqüência. Mc Kissock e col., em $1960^{14}$ e em $1965^{15}$, comparando grupos eqüivalentes de pacientes submetidos a tratamento clínico e cirúrgico concluiram não haver evidência de que a cirurgia fosse superior uma vez que ambos apresentavam uma taxa de mortalidade em torno de $40 \%$. A ligadura da carótida no pescoço, além de não afastar a possibilidade de novas roturas, freqüentemente pode se complicar de isquemia cerebral grave. A ligadura da artéria cerebral anterior que

* Professor Assistente de Clínica Neurológica, Neurocirurgiāo da Faculdade de Medicina da Universidade Feueral de Minas Gerais e Neurocirurgião do Hospital Felicio Rocho; ** Neurocirurgião do Hospital Felício Rocho; ** Residente em Neurocirurgia. 
supre o aneurisma tem sido utilizada por alguns $1,9,15,23$, com a mortalidade de 15 a $40 \%$. A proteção de aneurisma por acrílico ${ }^{5}$ e outras substâncias tem sido utilizada, embora haja casos de novas roturas, espasmo e necrose da parede arterial, além de defeitos lesivos sobre os nervos ópticos.

A maioria dos autores concorda em que o melhor tratamento dos aneurismas da artéria comunicante anterior consiste em craniotomia e clipagem do colo, isolando-o da circulação sem causar estenose nas artérias vizinhas e sem interferência com as artérias perfurantes $2,3,4,5,8,10,11,12,13,17,18,21$. Isso tem sido possivel atualmente, na maioria dos casos, utilizando-se o microscópio cirúrgico e instrumental especial, com uma mortalidade operatória baixa e cxcelentes resultados pós-operatórios ${ }^{13,25}$.

\section{MATERIAL E METODOS}

Logo depois da internação hospitalar os pacientes foram puncionados para comprovação da hemorragia subaracnóidea e colocados em repouso absoluto, em quarto escuro, sedados com barbitúricos e tratados com anti-hipertensivos caso apresentassem pressão arterial elevada. Difenilhidantoina foi prescrita com a idéia de se prevenir convulsões que poderiam causar nova rotura do aneurisma. Os pacientes com sinais de edema cerebral ou com sinais focais acentuados foram tratados com dexametasona intramuscular.

Quanto à gravidade classificamos nossos casos de acordo com os critérios de Botterel, modificados por Hunt e Hess ${ }^{11}$, em: grau 1 - assintomático ou cefaléia minima; grau 2 - cefaléia moderada a severa e rigidez de nuca, associadas a paresias de nervos cranianos; grau 3 - torpor, confusão mental e hemiparesia discreta; grau 4 - coma médio, hemiparesia acentuada a severa, distúrbios vegetativos; grau 5 - coma profundo, rigidez de decerebração, alterações vegetativas. Doenças sistêmicas graves como hipertensão arterial, diabete, pneumopatia crônica e espasmo intenso à angiografia colocam o caso, na escala, em tipo mais grave.

Quando admitidos em grau 1 ou 2 os pacientes foram logo submetidos a angiografia e operados precocemente. Os admitidos em grau 3, 4 e 5 foram submetidos a angiografia precoce e a tratamento médico até melhora ulterior; tais pacientes só são operados caso haja hematoma ameaçando sua vida. Neste caso, evacuamos o hematoma, tentando evitar o aneurisma. Nos dois últimos pacientes de nossa casuistica usamos o ácido epsilon aminocapróico (24 gramas/dia) ${ }^{19}$ no pré-operatório numa tentativa de prevenir nova rotura do aneurisma. Angiografia braquial direita retrógrada e carótida esquerda são realizadas em todos os pacientes para verificar possibilidade de aneurismas múltiplos.

O tratamento cirúrgico foi feito logo que os pacientes melhoraram até grau 1 ou imediatamente após o internamento nos que foram encaminhados já em boas condiçōes. A anestesia é induzida cuidadosamente evitando-se hipertensão, tosse ou agitação. Os pacientes são colocados na mesa cirúrgica e uma agulha de punção lombar é introduzida no espaço subaracnóideo para drenagem de líquido cefalorraqueano durante a intervenção. Faz-se também cateterismo vesical de demora. A cabeça do paciente é fixada em posição obliqua e ligeiramente elevada.

Fazemos, a seguir, craniotomia fronto-temporal baixa, do lado pelo qual se enche o aneurisma, e ressecção de terço lateral da asa de esfenóide. Logo no início da incisão, são administrados $500 \mathrm{ml}$ de Manitol a $20 \%$, gota a gota, por via endovenosa. Após a abertura da dura-mater, abre-se a drenagem liquórica e o microscópio 
cirúrgico * é colocado em posição; usamos objetiva de $300 \mathrm{~mm}$, ocular 20 , sendo o botão de magnificação colocado em x6 durante o início da dissecção e em x10-x16 durante a dissecção do colo do aneurisma. Veias de drenagem entre o polo temporal e a dura-mater são coaguladas e, com ajuda do afastador cerebral autostático (afastador Leyla-Yasargil), o lobo frontal é elevado.

A cisterna optoquiasmática é aberta, sendo identificados o nervo óptico, a carótida interna e a artéria cerebral anterior. A seguir, com uso do coagulador bipolar, microtesoura e microespátulas dissecamos a artéria cerebral anterior, tendo cuidado para não lesar os pequenos ramos perfurantes que são facilmente identificáveis com o uso do mícroscópio. Quando a base do aneurisma está próxima a pressão arterial deve ser baixada para $60 \mathrm{mmHg}$ com Arfonad.

Seguimos a técnica sugerida por Kempe ${ }^{12}$ e por Ark e Kempe ${ }^{24}$, fazendo uma ressecção cortical de giro nos aneurismas que apontam para baixo e para frente, e através da via sub-frontal nos que apontam para cima. Neste ponto a dissecção deve ser completa, devendo-se visualizar as porções proximal e distal de ambas cerebrais anteriores, uma vez que, ao ser colocado, o clip não deverá ocluir ou distorcer nenhum destes vasos. Evitamos a dissecção do fundo do aneurisma, colocando o clip no colo sem visualização de todo o saco aneurismático. Em apenas um caso não pudemos clipar com segurança tendo sido feita a exposição total do aneurisma e proteção com acrilico. Para clipagem temos utilizado os clips de Mayfield e Mc Fadden que são de vários formatos facilitando a reaplicação em caso de clipagem incorreta; o único problema é que a mola é grande e às vezes dificulta a visão no momento de sua aplicação.

Uma vez clipado o colo do aneurisma colocamos músculo macerado envolvendo a área e um pedaço de gelfoam embebido em cloridrato de papaverina numa tentativa de inlbir o espasmo pós-operatório. A pressão arterial é elevada para niveis normais e a hemostasia é completada com coagulador bipolar. A dura-mater é fechada em sutura contínua com mersilene e fixada ao periósteo com pontos de ancoragem. Fixa-se ainda a dura-mater através de um orifício feito no centro do retalho ósseo, colocando-se um clip metálico. O subcutâneo é fechado com pontos separados e a pele em sutura contínua com mononylon, sem uso de dreno. Nos casos em que se espera espasmo arterial, pelo estado pré-operatório e pelo trauma círúrgico, iniciamos, ná sala de cirurgia, a infusão de $500 \mathrm{ml}$ de Dextran.

As primeiras 24 horas de pós-operatório são passadas no centro de Tratamento Intensivo. No pós-operatório imediato mantemos o uso de Dexametasona, difenilhidantoina, antibióticos e hidratamos o paciente com aproximadamente $1500 \mathrm{ml}$ de solução glico-fisiológica (4:1), fazendo rigoroso balanço hídrico e tomando precaucōes para evitar hiponatremia ou hiperhidratação. Uma semana após a cirurgia realizamos angiografia de controle.

\section{R E S U T A D OS}

Conforme se vê no quadro 1 , operamos 8 pacientes com aneurismas de artéria comunicante anterior, sendo que dois (casos 3 e 7 ) apresentavam também aneurisma em outra localização. No caso 3 , clipamos o aneurisma de carótida e da comunicante anterior e, no caso 7, clipamos o aneurisma da cerebral média esquerda e protegemos com acrílico o da comunicante anterior. Como complicação operatória tivemos um caso de meningite purulenta (caso 7 ), que respondeu muito bem ao tratamento com antibióticos.

Com exceção do caso 8 , operado há poucas semanas e no qual a paciente está se recuperando do quadro mental de indiferença que já apresentava no pré-operatório, todos os outros puderam retornar às suas atividades normais. O caso 3 apresentou,

* Microscóplo binocular Zeiss tipo I. 
como seqüela, hemiparesia esquerda que, entretanto, não impede o paciente de exercer sua profissão de comerciante. Não houve mortalidade cirúrgica. Dos pacientes operados apenas um faleceu (caso 2), por traumatismo craniano; tratava-se de um engenheiro que, após a cirurgia, voltou às atividades normais que exerceu durante um ano, tendo falecido em desastre automobilístico.

A angiografia de controle mostrou que em um paciente (caso 8) havia enchimento de parte do aneurisma ( $20 \%$ proximal). Este paciente será submetido a novas angiografias de 6 em 6 meses para sabermos se esta parte do aneurisma terá tendência para aumentar ou para desaparecer ${ }^{3,5}$.

\begin{tabular}{|c|c|c|c|c|c|c|c|}
\hline $\mathrm{N}: \mathrm{O}$ & Nome & Sexo & $\begin{array}{l}\text { Idade } \\
\text { (anos) }\end{array}$ & Aneurisma & $\begin{array}{l}\text { Intervalo } \\
\text { entre a } \\
\text { rotura e a } \\
\text { cirurgia }\end{array}$ & $\begin{array}{c}\text { Pós- } \\
\text { operatório }\end{array}$ & $\begin{array}{c}\text { Seguimento } \\
\text { (até junho/1973) }\end{array}$ \\
\hline 1 & $\mathrm{ZR}$ & $\mathbf{F}$ & 35 & $\mathrm{CA}$ & 20 dias & normal & $\begin{array}{l}\text { paciente } \\
\text { trabalhando }\end{array}$ \\
\hline 2 & RVL & $\mathbf{M}$ & 27 & $\mathrm{CA}$ & 7 dias & normal & $\begin{array}{l}\text { trabalhou } 1 \text { ano, } \\
\text { falecendo por } \\
\text { traumatismo } \\
\text { cranianos }\end{array}$ \\
\hline 3 & DVC & M & 45 & CA+CAROT. & 40 dias & hemiparesia & $\begin{array}{l}\text { paciente } \\
\text { trabalhando }\end{array}$ \\
\hline 4 & $\mathrm{SCT}$ & $\mathrm{M}$ & 47 & $\mathrm{CA}$ & 7 dias & normal & $\begin{array}{l}\text { paciente } \\
\text { trabalhando }\end{array}$ \\
\hline 5 & $\mathrm{JCL}$ & M & 41 & $\mathrm{CA}$ & 15 dias & normal & $\begin{array}{l}\text { paciente } \\
\text { trabalhando }\end{array}$ \\
\hline 6 & ABS & M & 24 & $\mathrm{CA}$ & 5 dias & normal & $\begin{array}{l}\text { paciente } \\
\text { trabalhando }\end{array}$ \\
\hline 7 & JAP & M & 24 & $\mathrm{CA}+\mathrm{CM}$ & 15 dias & meningite & $\begin{array}{l}\text { paciente } \\
\text { trabalhando }\end{array}$ \\
\hline 8 & NMF & $\mathbf{F}$ & 58 & $\mathrm{CA}$ & 15 dias & normal & $\begin{array}{l}\text { paciente } \\
\text { em recuperação }\end{array}$ \\
\hline
\end{tabular}

Quadro 1 - Dados para identificação dos 8 pacientes operados e para avaliacão dos resultados: $C A=$ aneurisma de artéria comunicante anterior; CAROT. $=$ aneurisma de carótida interna; $C M=$ aneurisma de artéria cerebral média.

\section{COM EN TA R I O S}

A dissecção e clipagem de aneurismas da artéria comunicante anterior, tida como perigosa há alguns anos, pode ser realizada com segurança utilizando-se técnica microcirúrgica. A imagem angiográfica muitas vezes dá uma falsa idéia do aneurisma, não mostrando colo que, no entanto, pode ser individualizado para clipagem mediante dissecção sob amplificação. Na figura 1 vemos como um grande aneurisma aparentemente sem colo pode ser clipado sem prejuizo para a circulação da artéria cerebral. 

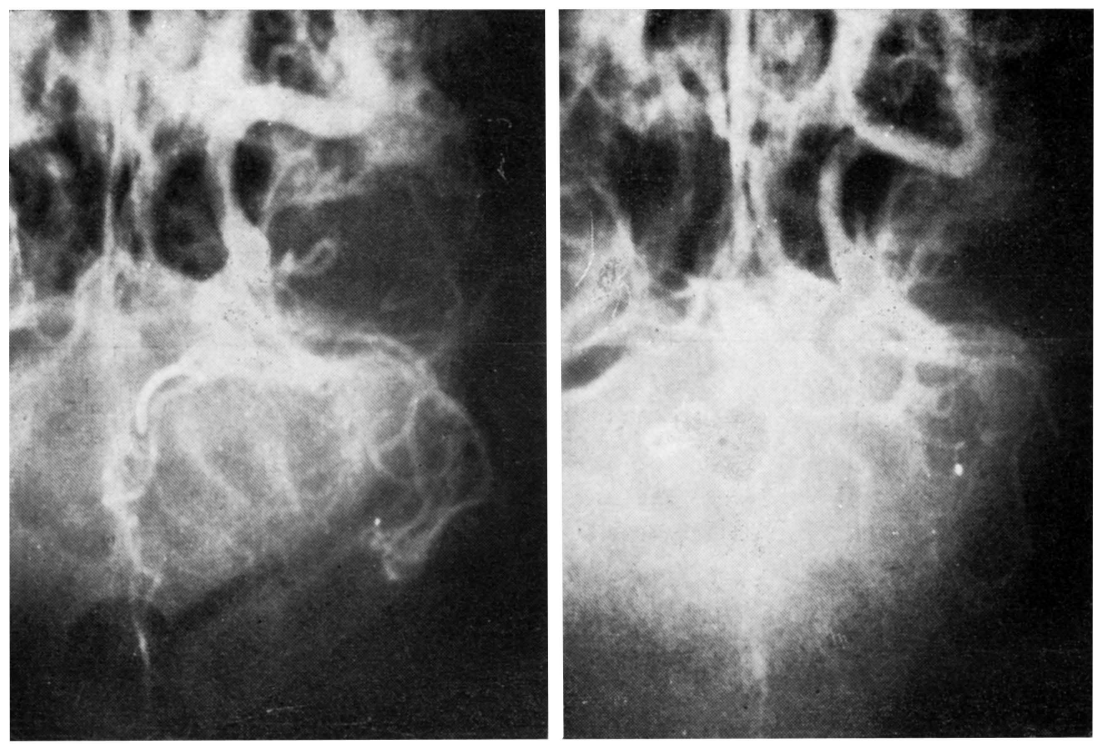

Fig. 1 - Angiografias carotídeas pré e pós-operatória do paciente S.C.T. (caso 4): o colo do aneurisma foi bem individualizado mediante dissecção microscópica, apesar de nũo ser visível na angiografia pré-operatória.

Krayenbühl e col. ${ }^{13}$ aconselham o uso de coagulação bipolar no colo, provocando a sua retração e facilitando a clipagem. Na literatura consultada notamos que os resultados variam muito com a técnica de tratamento empregada, com a condição pré-operatória e com o temfo decorrente entre a hemorragia e a intervenção cirúrgica. Quanto maior o intervalo entre a rotura do aneurisma e o ato cirúrgico melhores serão os resultados. Isto tem levado alguns autores a aguardar duas semanas após a rotura para operar seus pacientes. Entretanto alguns pacientes podem sofrer nova hemorragia neste periodo de espera; estatisticamente a segunda rotura ocorre com maior freqüência neste período ${ }^{20}$. Assim, nossa conduta tem sido a de operar o mais cedo possivel os pacientes com sintomatologia de grau 1 e 2. O ácido epsilon aminocapróico parece diminuir a incidência de novas roturas e o usamos associado a medicamentos hipotensores durante o período de espera da melhoria de condiçōes dos pacientes com sintomatologia de grau 3, 4 e 5 .

Sem preocupação de fazer uma revisão completa da literatura, procuramos estabelecer a mortalidade cirúrgica referida por diversos autores que empregaram técnicas diversas de tratamento. Ahmed e Sedzimir ${ }^{1}$ submeteram 60 pacientes a ligadura proximal da artéria cerebral anterior com mortalidade de $15 \%$; Tindall e col. ${ }^{23}$ empregaram a mesma técnica além de ligadura da carótida contralateral em 24 casos com mortalidade de $16,7 \%$; Hamby ${ }^{8}$ realizou clipagem em 38 casos com mortalidade de $16 \%$; Pool ${ }^{18}$ operou 60 pacientes 
por via frontal interhemisférica, com mortalidade de $7 \%$; Sampaio ${ }^{21}$ fez clipagem em 14 pacientes com mortalidade de $28 \%$; Krayenbühl e col. ${ }^{13}$ operaram 95 pacientes pela técnica microcirúrgica com mortalidade de $3 \%$, sendo que entre os casos grau 1 e grau 2 não houve óbito cirúrgico.

$\mathrm{Na}$ presente série de 8 pacientes não houve mortalidade cirúrgica, $88 \%$ dos pacientes voltando a trabalhar normalmente.

\section{R E S M O}

Os autores descrevem o tratamento microcirúrgico realizado em oito pacientes que tiveram hemorragia subaracnóidea espontânea por rotura de aneurisma da artéria comunicante anterior. A conduta clínica pré-operatória é apresentada sendo dada ênfase às condições do paciente antes do ato cirúrgico. A técnica cirúrgica é descrita em detalhe, sendo salientado o uso do microscópio binocular. Dos 8 pacientes, dois $(25 \%)$ tinham mais de um aneurisma que foram tratados durante a mesma operação. A única complicação cirúrgica foi meningite pós-operatória em um paciente; esta complicação cedeu completamente ao tratamento. Após a operação $88 \%$ dos pacientes retornaram ao seu trabalho, não tendo havido óbito em virtude do tratamento.

\section{S U M M A R Y}

Microsurgical treatment of anterior communicating artery aneurysms.

The surgical treatment of eight patients who had spontaneous subarachnoid hemorrhage from ruptured anterior communicating aneurysms is reported. The pre-operatory treatment is described, with emphasis to the patients condition before surgery. The surgical technique is described with special reference to the use of binocular microscope. Two patients $(25 \%)$ had multiple aneurysms which were treated at the same operation. Meningitis was a complication on one patient but subsided with antibiotic treatment. After the operation $88 \%$ of the patients returned to their primitive jobs and there was no death related to the surgical treatment. Comments on other procedures for the surgical treatment of anterior communicating aneurysms are made.

REFER È N C I S

1. AHMED, R. H. \& SEDZIMIR, C. B. - Ruptured anterior communicating aneurysms. A comparison of medical and specific surgical treatment. J. Neurosurg. $26: 213,1967$.

2. ALMEIDA, G. M.; TILBERY, C. P. \& GAGLIARDi FERREIRA, L. C. - Tratamento dos aneurismas intracranianos. Rev. Paul. Med. 77:193, 1971.

3. BONNAL, J. \& STEVENAERT, A. - Thrombosis of intracranial aneurysms of the circle of Willis after incomplete obliteration by clip or ligature across the neck. J. Neurosurg. 30:158, 1969.

4. DAVID, M.; PERTUIZET, B. \& GUYOT, J. F. - La cure radicale des anérysmes de la communicant antérieure sans résection cérébrale ni hypothermie dans le malade conscient. Présse Méd. 76:1467, 1968. 
5. DRAKE, C. G. \& VANDERLINDERS, R. G. - The late consequences of incomplete surgical treatment of cerebral aneurysms. J. Neurosurg. 27:226, 1967.

6. DUTTON, J. - Acrylic investment of intracranial aneurysms. A report of 12 years experience. J. Neurosurg. 31:652, 1969.

7. GRAF, C. J. - Prognosis for patient with non surgically treated aneurysms. Analysis of the cooperative study of intracranial aneurysms and subarachnoid hemorhage. J. Neurosurg. 35:438, 1971.

8. HAMBY, W. - Remarks Concerning Intracranial Aneurysmal Surgery. Proc. Congress of Neurological Surgeons. Williams a. Wilkins Co., Baltimore, 1970.

9. HUGENHOLTZ, H. \& MORLEY, T. P. - The results of proximal anterior cerebral artery oclusion for anterior communicating aneurysms. J. Neurosurg. $37: 65,1972$.

10. HUNT, W. E.; MEAGHER, J. N. \& BARNES, J. E. - The management of intracranial aneurysms. J. Neurosurg. 19:34, 1962.

11. HUNT, 'W. E. \& HESS, R. - Surgical risk as related to time of intervention in the repair of intracranial aneurysms. J. Neurosurg. 28:14, 1968.

12. KEMPE, L. C. - Operative Neurosurgery. Springer Verlag New York Inc., 1968.

13. KRAYENBUHL, H. A.; YASARGIL, M. G.; FLAMM, E. S. \& TEW, J. M. Microsurgical treatment of intracranial saccular aneurysms. J. Neurosurg. 37: $678,1972$.

14. Mc KISSOCK, W.; PAINE, K. W. E. \& WALSH, L. S. - An analysis of the results of treatment of ruptured intracranial aneurysms. Report of 772 consecutive cases. J. Neurosurg. 17:762, 1960.

15. Mc KISSOCK, W.; RICHARDSON, H. \& WALSH, L. S. - Anterior communicating aneurysms. A trial of conservative and surgical treatment. Lancet 1:873, 1965.

16. Mc MURTRY III, J. G.; POOL, J. L. \& NOVA H. - The use of Rheomacrodex in the surgery of intracranial aneurysms. J. Neurosurg. 26:218, 1967.

17. PAUL, R. L. \& ARNOLD, J. G. - Operative factors influencing mortality in intracranial aneurysms surgery. Analysis of 186 consecutive cases. J. Neurosurg. 32:280, 1970.

18. POOL, J. L. - Bifrontal craniotomy for anterior communicating artery aneurysm. J. Neurosurg. 36:212, 1972.

19. RANSOHOFF, J.; GOODGOLD, H. \& BENJAMIN, M. V. - The operative management of patients with ruptured intracranial aneurysms. J. Neurosurg. $36: 523,1972$.

20. SAHS, A. L.; PERRET, G. E.; LOCKSLEY, H. B. \& NISHIOKA, H. - Intracranial Aneurysms and Subarachnoid Hemorrhage. A Cooperative Study. J. B. Lippincott Co., Philadelphia, 1969.

21. SAMPaIO, P. - Aneurismas da artéria comunicante anterior. Consideraçōes sobre 14 casos operados. Arq. de Neuro-Psiquiat. (São Paulo) 28:367, 1970.

22. STEhbens, w. E. - Pathology of the Cerebral Blood Vessels. C. V. Mosby, Saint Louis, 1972.

23. TINDALL, G. T.; KAPP, J.; ADAM, G. L. \& ROBINSON, S. C. - A combined technique for treating certain aneurysms of the anterior communicating artery. J. Neurosurg. 33:41, 1970 .

24. VANDER ARK, G. D. \& KEMPE, L. C. - Classification of anterior communicating aneuryms as a basis for surgical approach. J. Neurosurg. 32:300, 1970.

25. YASARGIL, M. G. - Microsurgery Applied to Neurosurgery. Georg Thieme Verlag, Stuttgart, 1969. 